\title{
Jordan Higher Bi- Homomorphism and Co- Jordan Higher Bi- Homomorphism on Banach Algebra
}

\author{
Rajaa C. Shaheen
}

Mathematics Department, Education College, University of Al-Qadisiyah, Al-Qadisiyah, Iraq.

E-mail: Rajaa.chaffat@qu.edu.iq

ORCID ID: https://orcid.org/0000-0003-2783-9298

Received 8/1/2020, Accepted 13/10/2020, Published 30/3/2021

This work is licensed under a Creative Commons Attribution 4.0 International License.

\begin{abstract}
The concepts of higher Bi- homomorphism and Jordan higher Bi- homomorphism have been introduced and studied the relation between Jordan and ordinary higher Bi- homomorphism also the concepts of Cohigher $\mathrm{Bi}$ - homomorphism and $\mathrm{Co}$ - Jordan higher $\mathrm{Bi}$ - homomorphism introduced and the relation between them in Banach algebra have also been studied.
\end{abstract}

Keywords: Banach algebra, Co-Homomorphism ,Homomorphism, Jordan Homomorphism, Jordan Map.

\section{Introduction:}

Let $\mathrm{H}$ and $\mathrm{G}$ are complexes Banach algebras and let $\xi: H \rightarrow G$ be an additive map. Then $\xi$ is said $n$ homomorphism if for every $a_{1}, a_{2}, \ldots a_{n} \in H$ (1)

$$
\xi\left(a_{1}, a_{2}, \ldots, a_{n}\right)=\xi\left(a_{1}\right) \xi\left(a_{2}\right) \ldots \xi\left(a_{n}\right)
$$

Hejazian Sh. and Mirzavaziri M in (1) studied the concept of $n$-homomorphism on complex algebra. 2 - homomorphism being homomorphism, on this sense .We indicate to Bracic J., and Moslehian M.S in (2) on 3- homomorphism on certain attributes . Zelazko $\mathrm{W}$ in (3) introduced the definition of an $\mathrm{m}$ Jordan homomorphism. An additive map $\xi$ between $A$ and $B$ which are Banach algebras is said to be $m$ Jordan homomorphism(for short $\mathrm{m}-\mathrm{JH}$ ) if

$$
\xi\left(x^{m}\right)=(\xi(x))^{m}, \quad \text { for all } x \in A
$$

A 2-Jordan homomorphism is said to be Jordan homomorphism. Every $m$ - homomorphism is $m$ Jordan homomorphism. But generally the converse is false and it is true with some conditions. Zelazko $\mathrm{W}$ in (4) shows from Banach algebra into commutative semi simple Banach algebra that every Jordan homomorphism is a homomorphism . Zivari $A$ in (5) refers to the reader to characterizations of 3- Jordan Homomorphism. Zelazko W in (3) shows that every m-Jordan homomorphism between commutative Banach algebras is an $\mathrm{m}$ homomorphism for $\in\{2,3,4\}$. An G. , Ding Y. and Li J in (6)and Liu L in(7)some results about Jordan centralizers and Jordan derivations done.
In this article, let $U=A \times B$. Then $U$ with multiplication

$(a, b)(x, y)=(a x, b y),(a, b),(x, y) \in \mathcal{U}$

is Banach algebra. It is unital if and only if $A$ and $B$ are unital and it is commutative if and only if $A$ and $B$ are commutative and semi simple if its radical is zero also refer by $e$ to the unital element of $\mathrm{A}$ and B. Suppose $D$ is complex Banach algebras. Bilinear map is a function $\xi: \mathcal{U} \rightarrow D$ such that for any $a \in A$ the map $b \rightarrow \xi(a, b)$ is a map from $\mathcal{U}$ to $D$, and for any $b \in \mathcal{U}$ the map $a \mapsto \xi(a, b)$ is additive from $A$ to $D$.

Zivari A in(8) defined Jordan (resp,.Bihomomorphism)as follows

A bi- additive mapping $\delta$ is said $\mathrm{Bi}$ homomorphism if for every $(a, b),(x, y) \epsilon A \times B$,

$\delta(a x, b y)=\delta(a, b) \delta(x, y)$

And it is called Bi- Jordan homomorphism if $\delta\left(a^{2}, b^{2}\right)=(\delta(a, b))^{2}$

Bi- Homomorphism be Jordan Bi- Homomorphism. In general the converse is not true Zivari A in(8) shows that the converse holds under some conditions .In this article, the concepts of Jordan (higher $\mathrm{Bi}$ - homomorphism) and the relation between them have been introduced and studied. An additive map $\delta$ between $A$ and $B$ which are Banach algebra is said to Co- homomorphism if $\delta(x y)=$ $-\delta(x) \delta(y), x, y \in$ A.and it is called a Co- Jordan homomorphism $\delta\left(x^{2}\right)=-(\delta(x))^{2}$ for all $x \in A$, Zivari A in (5) studied the relation between CoJordan homomorphism and Co- homomorphism under certain condition. In this article, the concepts 
of $\mathrm{Co}-\mathrm{Bi}$ - higher homomorphism and $\mathrm{Co}-\mathrm{Bi}$ Jordan higher homomorphism and the relation between them have been introduced and studied .

\section{Jordan Higher Bi- Homomorphism Definition 2.1:}

Let $=\left\{\delta_{i}\right\}_{i \in N}, N$ is the natural number, be the family of bi- Linear map $\delta_{i}=R \times R \rightarrow R$, $(a, b),(x, y) \epsilon U, U=R \times R$ is called higher bihomomorphism $\delta_{n}(a x, b y)=\sum_{i=1}^{n} \delta_{i}(a, b) \delta_{i}(x, y)$ and is said Jordan higher Bihomomorphism(JHBH, for short) if $\delta_{n}\left(a^{2}, b^{2}\right)=\sum_{i=1}^{n}\left(\delta_{i}(a, b)\right)^{2}, a, b \in U$.

Lemma 2.2: Suppose that $\delta: U \rightarrow R$ is a Jordan higher Bi-homomorphism. Then

1. $\delta_{n}\left(x y+y x, b^{2}\right)=\sum_{i=1}^{n} \delta_{i}\left(x y, b^{2}\right) \delta_{i}\left(y x, b^{2}\right)$

2. $\delta_{n}\left(a^{2}, x y+y x\right)=\sum_{i=1}^{n} \delta_{i}(a, \mathrm{xy}) \delta_{i}(a, y x)$

Proof: it is straight forward

Lemma 2.3: Let $\delta$ be a Jordan higher $\mathrm{Bi}$ homomorphism. If $U$ commutative and unital, then

1. $\delta_{n}(a x, e)=\sum_{i=1}^{n} \delta_{i}(a, e) \delta_{i}(x, e)$

2. $\delta_{n}(e, b y)=\sum_{i=1}^{n} \delta_{i}(e, b) \delta_{i}(e, y)$.

Proof: From lemma 2.2, if suppose that $\mathrm{y}=\mathrm{a}$ and use commutativity .

Lemma 2.4: Let $U$ be unital and let $\delta$ be a nontrivial and non-empty family of Jordan higher $\mathrm{Bi}$ homomorphism(for short JHBH). Then $\delta(e, e) \neq 0$.

Proof: Since $\delta$ is a Jordan higher $\mathrm{Bi}$ homomorphism. Then

$\forall(a, b) \epsilon U$, we get

$\delta\left(a^{2}, b^{2}\right)=\sum_{i=1}^{n}\left(\delta_{i}(a, b)\right)^{2}$

Replacing $a$ by $x+e$, by $y+e$ in (4), Gives

$\delta_{n}\left(x^{2}+2 x+e, y^{2}+2 y+e\right)=$

$\sum_{i=1}^{n}\left(\delta_{i}(x+e, y+e)\right)^{2}$

Suppose that $\delta_{n}(e, e)=0$. Then by lemma 2.3

$\delta_{n}(x, e)=\delta_{n}(e, y)=0 \quad \forall(x, y) \epsilon U$

(6)

It follows from (5) and (6) that

$\delta_{n}(2 x, 2 y)+\delta_{n}\left(2 x, y^{2}\right)+\delta_{n}\left(x^{2}, 2 y\right)=0$

(7)

For all $(x, y) \in U$. By lemma2.3

$\delta_{n}\left(2 x, y^{2}\right)=\sum_{i=1}^{n} 2 \delta_{i}(x, y) \delta_{i}(e, y)=0$

$\left.\delta_{n}\left(x^{2}, 2 y\right)=\sum_{i=1}^{n} 2 \delta_{i}(x, e) \delta_{i}(x, y)=0\right\}$

(8)

Then by (7) and ( 8), we get

$\delta_{n}(x, y)=0 \quad \forall(x, y) \epsilon U$, which is contradicted with $\delta$ non-trivial.

Theorem 2.5: Let $U$ commutative and unital and $\delta$ be a Jordan Higher Bi-homomorphism from $U$ into a semi simple Banach commutative algebra $D$. Then $\delta$ is higher than Bi-homomorphism.

Proof: Let $D=\mathbb{C}$ and let $\delta=\left\{\delta_{i}\right\}_{i \in N}, \delta_{i}: U \rightarrow \mathbb{C}$ be a Jordan higher Bi-homomorphism. So for every $(a, b) \epsilon U$, we get

$\delta_{n}\left(a^{2}, b^{2}\right)=\sum_{i=1}^{n}\left(\delta_{i}(a, b)\right)^{2}$

(9)
Replacing $a$ by $x+e$, and $b$ by $y+e$ in(9)

$\delta_{n}\left(a^{2}, b^{2}\right)=\sum_{i=1}^{n}\left(\delta_{i}(a, b)\right)^{2}$

$\delta_{n}\left(x^{2}+2 x+e, y^{2}+2 y+e\right)=\sum_{i=1}^{n}\left(\delta_{i}(x+\right.$

$e, y+e))^{2}$

By lemma $2.4, \delta_{n}(e, e) \neq 0$, so ( 10$)$ gives

$\delta_{n}(e, e)=1$

From lemma 2.2, we get

$\left.\delta_{n}\left(2 x, y^{2}\right)=2 \sum_{i=1}^{n} \delta_{i}(x, y) \delta_{i}(e, y)\right\}$

$\left.\delta_{n}\left(x^{2}, 2 y\right)=2 \sum_{i=1}^{n} \delta_{i}(x, e) \delta_{i}(x, y)\right\}$

(12)

Thus by (10), (11) and (12), we get

$\delta_{n}(x, y)=\sum_{i=1}^{n} \delta_{i}(x, e) \delta_{i}(e, y)$ for all $(x, y) \in U$ (13)

Replace $x$ by $a x$ and $y$ by by in (13) gives

$\delta_{n}(a x, b y)=\sum_{i=1}^{n} \delta_{i}(a x, e) \delta_{i}(e, b y)$

By (14) and lemma 2.4, we get

$\delta_{n}(a, b) \delta_{n}(x, y)=\sum_{i=1}^{n} \delta_{i}(a, b) \delta_{i}(x, y)=$

$\sum_{i=1}^{n}\left[\sum_{i=1}^{n} \delta_{i}(a, e) \delta_{i}(x, e)\right] .\left[\sum_{i=1}^{n} \delta_{i}(e, b) \delta_{i}(e, y)\right]$

$=\sum_{i=1}^{n} \delta_{i}(a x, e) \delta_{i}(e, b y)$

By (14) and (15)

$\delta_{n}(a x, b y)=\sum_{i=1}^{n} \delta_{i}(a, b) \delta_{i}(x, y)$

For all $(a, b),(x, y) \epsilon U$ and so $\delta$ is a Bi- higher homomorphism.

Suppose $\mathcal{D}$ is commutative semi simple, $\mathcal{M}(\mathcal{D})$ be maximal ideal space of $\mathcal{D}$, with each $\mathrm{f} \in \mathcal{M}(\mathcal{D})$, associate a function $\delta_{f}: U \rightarrow \mathbb{C}$ defined by

$\delta_{f}(a, b)=f(\delta(a, b)),(a, b) \epsilon U$

Pick $\mathrm{f} \in \mathcal{M}(\mathcal{D})$ arbitrary. $\delta_{f}$ is a Jordan Higher Bihomomorphism so it is a higher $\mathrm{Bi}$ homomorphism. So from definition of $\delta_{n f}$, we get

$$
\begin{aligned}
f\left(\delta_{n}(a x, b y)\right) & =f\left(\sum_{i=1}^{n} \delta_{i}(a, b) \delta_{i}(x, y)\right. \\
& =f\left(\sum_{i=1}^{n} \delta_{i}(a, b)\right) f\left(\sum_{i=1}^{n} \delta_{i}(x, y)\right) \\
& =f\left(\delta_{n}(a, b)\right) f\left(\delta_{n}(x, y)\right)
\end{aligned}
$$

Since $f \in \mathcal{M}(\mathcal{D})$ was arbitrary and $D$ assuming to be semi simple, we obtain $\delta_{n}(a x, b y)=\sum_{i=1}^{n} \delta_{i}(a, b) \delta_{i}(x, y)$

$\forall(x, y),(a, b) \in U$

\section{Co- Jordan Higher bi- Homomorphism}

Definition 3.1: A family $\delta=\left\{\delta_{i}\right\}, \delta_{i}: A \times A \rightarrow B$ where $A$ and $B$ are Banach algebra is called a Co Bi-higher homomorphism if $\delta_{n}(a b, c d)=$ $-\sum_{i=1}^{n} \delta_{i}(a, c) \delta_{i}(b, d)$

And is called a Co- Jordan higher Bihomomorphism if $a=b, c=d$.

Theorem 3.2: Suppose that $A$ is a Banach algebra need not to be commutative. Then each Co- Jordan higher Bi-homomorphism a Co- higher $\mathrm{Bi}$ homomorphism.

Proof: Suppose that $\delta$ is a Co- Jordan higher Bihomomorphism,

So $\delta_{n}\left(a^{2}, c^{2}\right)=-\sum_{i=1}^{n} \delta_{i}(a, c) \delta_{i}(a, c) \quad \forall a, c \in A \quad$, replaced $a$ by $a+b$ gives $\delta_{n}\left(a b+b a, c^{2}\right)=$ $-2 \sum_{i=1}^{n} \delta_{i}\left(a, c^{2}\right) \delta_{i}\left(b, c^{2}\right) \forall a, b, c \in A$

then by (18), 
$2 \delta_{n}\left(a b a, c^{3}\right)=\delta_{n}((a b+b a) a+a(a b+$ $\left.b a), c^{3}\right)-\delta_{n}\left(a^{2} b+b a^{2}, c^{3}\right)$

$=-2\left(\sum_{i=1}^{n} \delta\left({ }_{i}\left(a b+b a, c^{2}\right) \delta_{i}(a, c)-\right.\right.$ $\left.\delta_{i}\left(a^{2}, c^{2}\right) \delta_{i}(b, c)\right)$

$=$

$-2\left[-2 \sum_{i=1}^{n} \delta_{i}(a, c) \delta_{i}(b, c)+\right.$

$\left.\left.\delta_{i}(a, c) \delta_{i}(a, c)\right] \delta_{i}(b, c)\right)$

$2 \sum_{i=1}^{n} \delta_{i}(a, c) \delta_{i}(a, c) \delta_{i}(b, c)$, therefore

$\delta_{n}\left(a b a, c^{3}\right)=$

$\sum_{i=1}^{n} \delta_{i}(a, c) \delta_{i}(a, c) \delta_{i}(b, c) \quad \forall a, b, c \in A$

Let $a$ and $b \in A$ and put

$2 t=\delta_{n}\left(a b-b a, c^{2}\right)$

It is clear from (18) and (20) that

$\delta_{n}\left(a b, c^{2}\right)-t=-\sum_{i=1}^{n} \delta_{i}(a, c) \delta_{i}(b, c)$

$\delta_{n}\left(b a, c^{2}\right)+t=-\sum_{i=1}^{n} \delta_{i}(a, c) \delta_{i}(b, c)$

By (19)-(21)

$4 t^{2}=\left(\delta_{n}\left(a b-b a, c^{2}\right)\right)^{2}=\delta_{n}\left(\left((a b)^{2}+\right.\right.$

$\left.(b a)^{2}-a b^{2} a-b a^{2} b\right),\left(c^{2}\right)^{2}$

$=$

$\delta_{n}\left((a b)^{2}, c^{4}\right)+\delta_{n}\left((b a)^{2}, c^{4}\right)-\delta_{n}\left(a b^{2} a, c^{4}\right)-$

$\delta_{n}\left(b a^{2} b, c^{4}\right)$

$=\left[t-\sum_{i=1}^{n} \delta_{i}(a, c) \delta_{i}(b, c)\right]^{2}+[-t-$

$\left.\sum_{i=1}^{n} \delta_{i}(a, c) \delta_{i}(b, c)\right]^{2}-$

$\left[2 \sum_{i=1}^{n}\left(\delta_{i}(a, c)\right)^{2}\left(\delta_{i}(b, c)\right)^{2}\right.$

$=2 t^{2}$

Then $t=0$ which proves that $\delta_{n}\left(a b, c^{2}\right)=$ $\delta_{n}\left(b a, c^{2}\right)$

Therefore, by

$\delta_{n}\left(a b, c^{2}\right)=-\sum_{i=1}^{n} \delta_{i}(a, c) \delta_{i}(b, c)$.

\section{Conclusion:}

In this article, Jordan higher Bi- homomorphism on Banach algebra is higher than $\mathrm{Bi}$ homomorphism also Jordan Co- higher $\mathrm{Bi}$ - homomorphism on Banach algebra is Co- higher $\mathrm{Bi}$ - homomorphism under appropriate conditions.

\section{Author's declaration:}

- Conflicts of Interest: None.

- Ethical Clearance: The project was approved by the local ethical committee in University of AlQadisiyah.

\section{References:}

1. Hejazian Sh, Mirzavaziri M, Moslehian MS. nHomomorphisms. Bulletin of the Iran. Math. Soc. 2005 Mar.; 31( 1):13-23.

2. Bracic J, Moslehian M S. On Automatic Continuity of 3-Homomorphisms on Banach Algebras .Bull. Malays. Math. Sci. Soc. 2007; Second Series , 30(2): 195-200.

3. Eshaghi M. n-Jordan homomorphisms. Bull.Aus. Math.Soc. 2009 Aug.;80(1):159-164.Availablefrom DOI: https://doi.org/10.1017/S000497270900032X.

4. Zelazko W. A characterization of multiplicative linear functionals in complex Banach algebras. Studia Math. 1968; 30:83-85.

5. Zivari A. A characterization of 3-Jordan homomorphism on Banach algebras. Bull. Aus. Math. Soc. 2016 April , 93( 2): 301-306. Available from DOI: https://doi.org/10.1017/S0004972715001057X

6. An G, Ding Y, Li J. Characterizations of Jordan left derivations on some algebras. Banach .J. Math. Anal., 2016, 10(3): 466-481.

7. Liu L. On Jordan centralizers of triangular algebras. Ban. J. Math. Anal., 2016; 10( 2): 223-234.

8. Zivari A. A characterization of Bi-Jordan homomorphism on Banach algebras. Int. J. Ana., 2017;2017Article ID4206871:5pages.

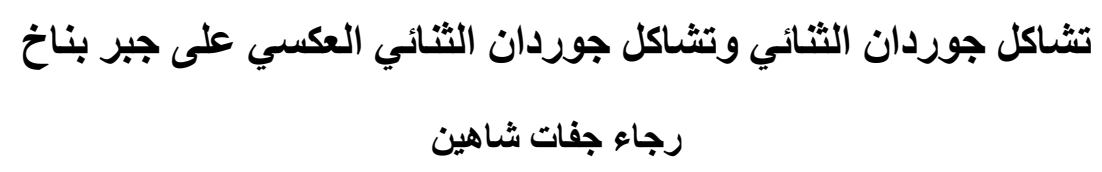

قسم الرياضيات، كلية التربية، جامعة القادية، القادسية، العراق.

قدمت مفاهيم التشاكل الثنائي من الرتب العليا وتشاكل جوردان الثنائي من الرتب العليا ودرست العلاقة بين تنشاكل جوردان الثنائي

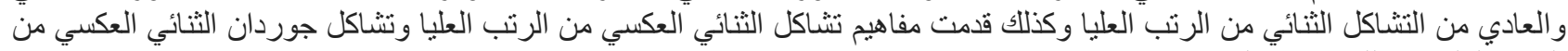

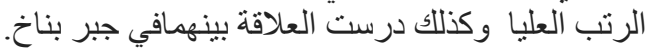

الكلمات المفتاحية: التشاكل، التشاكل العكسي، تطبيق جوردان، تشاكل جوردان، جبر بناخ. 